University of Nebraska - Lincoln

DigitalCommons@University of Nebraska - Lincoln

$4-1-2006$

\title{
Structures and relative stability of medium-sized silicon clusters. V. Low-lying endohedral fullerenelike clusters $\mathrm{Si}_{31}-\mathrm{Si}_{40}$ and $\mathrm{Si}_{45}$
}

Soohaeng Yoo

University of Nebraska-Lincoln

N. Shao

University of Nebraska-Lincoln

C. Koehler

Theoretische Physik, Universität-GH Paderborn, D-33098 Paderborn, Germany

T. Fraunhaum

Theoretische Physik, Universität-GH Paderborn, D-33098 Paderborn, Germany

Xiao Cheng Zeng

University of Nebraska-Lincoln, xzeng1@unl.edu

Follow this and additional works at: https://digitalcommons.unl.edu/chemzeng

Part of the Chemistry Commons

Yoo, Soohaeng; Shao, N.; Koehler, C.; Fraunhaum, T.; and Zeng, Xiao Cheng, "Structures and relative stability of medium-sized silicon clusters. V. Low-lying endohedral fullerenelike clusters $\mathrm{Si}_{31}-\mathrm{Si}_{40}$ and Si 45 " (2006). Xiao Cheng Zeng Publications. 9.

https://digitalcommons.unl.edu/chemzeng/9

This Article is brought to you for free and open access by the Published Research - Department of Chemistry at DigitalCommons@University of Nebraska - Lincoln. It has been accepted for inclusion in Xiao Cheng Zeng Publications by an authorized administrator of DigitalCommons@University of Nebraska - Lincoln. 


\title{
Structures and relative stability of medium-sized silicon clusters. V. Low-lying endohedral fullerenelike clusters $\mathbf{S i}_{31}-\mathbf{S i}_{40}$ and $\mathbf{S i}_{45}$
}

\author{
Soohaeng Yoo and N. Shao \\ Department of Chemistry, University of Nebraska-Lincoln, Lincoln, Nebraska 68588 and Center \\ for Materials Research and Analysis, University of Nebraska-Lincoln, Lincoln, Nebraska 68588 \\ C. Koehler and T. Fraunhaum \\ Theoretische Physik, Universität-GH Paderborn, D-33098 Paderborn, Germany \\ X. C. Zeng ${ }^{\text {a) }}$ \\ Department of Chemistry, University of Nebraska-Lincoln, Lincoln, Nebraska 68588 and Center \\ for Materials Research and Analysis, University of Nebraska-Lincoln, Lincoln, Nebraska
}

(Received 1 February 2006; accepted 7 March 2006; published online 27 April 2006)

\begin{abstract}
We have performed unconstrained search for low-lying structures of medium-sized silicon clusters $\mathrm{Si}_{31}-\mathrm{Si}_{40}$ and $\mathrm{Si}_{45}$, by means of the minimum-hopping global optimization method coupled with a density-functional based tight-binding model of silicon. Subsequent geometric optimization by using density-functional theory with the PBE, BLYP, and B3LYP functionals was carried out to determine the relative stability of various candidate low-lying silicon clusters obtained from the unconstrained search. The low-lying characteristics of these clusters can be affirmed by comparing the binding energies per atom of these clusters with previously determined lowest-energy clusters $\left(\mathrm{Si}_{n}\right)$ in the size range of $21 \leqslant n \leqslant 30$. In view of the fact that there exist numerous low-lying "endohedral fullerenelike" isomers for each size in the range $30 \leqslant n \leqslant 40$, we used the homologue carbon-fullerene cage to classify different families of isomers. This structural classification allows us to focus on generic features of various isomers and to group many apparently different isomers into a single family. In addition, we report a new family of low-lying clusters which have "Y-shaped three-arm" structures. Isomers in this "handmade" family can be energetically competitive as the endohedral fullerene isomers when the total energies are calculated with the BLYP or B3LYP functional. @ 2006 American Institute of Physics. [DOI: 10.1063/1.2191494]
\end{abstract}

\section{INTRODUCTION}

Ion mobility experiments by Jarrold and co-workers ${ }^{1-5}$ have revealed an important structural transition, namely, the transition from prolate to near-spherical structures for silicon cation $\left(\mathrm{Si}_{n}{ }^{+}\right)$and anion $\left(\mathrm{Si}_{n}{ }^{-}\right)$clusters in the size range of $24<n<30$. Recently, this structural transition has also been confirmed by a combined experimental/simulated anion photoelectron spectroscopy study ${ }^{6}$ as well as by an unconstrained global search for the cation clusters ${ }^{7}$ as well as a constrained search for the neutral clusters. ${ }^{8}$ In addition, the anion photoelectron spectroscopy experiment/simulation strongly supports that many near-spherical clusters in the size range of $30 \leqslant n \leqslant 40$ have outer cages homologue to the carbon-fullerene cages. ${ }^{9-16}$

It is well known that as the sizes of clusters increase, determination of true global minima becomes increasingly challenging because of the growing number of low-lying isomers. In papers III (Ref. 17) and IV (Ref. 8) of this series, we also showed that if the total energy of clusters are calculated using the density-functional theory (DFT), the determination of global minima can sometimes depend on the functional (e.g., PBE or BLYP) selected, particularly when there are several low-lying isomers having energy very close to each other (typically with energy differences less than $0.1 \mathrm{eV}$ ). In

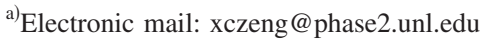

the latter case, high-level $a b$ initio calculation (e.g., calculation based on the coupled-cluster method) with a large basis set will be required to determine the true global minimum. ${ }^{18}$ However, with current computer facility, the high-level coupled-cluster calculation with very large basis sets is still impractical for medium-sized silicon clusters beyond $n=16$.

In view of the lack of experiments for neutral silicon clusters and the impracticalness of high-level ab initio quantum-chemistry calculation for medium-sized clusters, in our previous study of low-lying clusters in the size range of $20 \leqslant n \leqslant 30$ (paper IV) we proposed to give more attention to the generic structural features of low-lying clusters as a function of the size, rather than to focus on the prediction of a single (true) global-minimum structure. In fact we attempted to characterize different families of low-lying clusters on the basis of different building blocks (or motifs) of the clusters. The idea of motif-based characterization of silicon clusters was originally put forth by Ho and co-workers ${ }^{19,20}$ who discovered that many low-lying (including the lowest-energy) clusters in the size range of $12 \leqslant n \leqslant 18$ contain the tricapped-trigonal-prism (TTP) $\mathrm{Si}_{9}$ motif. Recently, we showed, based on previous unconstrained ${ }^{21}$ and constrained ${ }^{22}$ searches, that another structural motif can be a generic one for the low-lying clusters in the size range of $16 \leqslant n \leqslant 22$, that is, the six/six (or $\mathrm{Si}_{6} / \mathrm{Si}_{6}$ ) motif. Here, the first $\mathrm{Si}_{6}$ refers to the puckered hexagonal ring unit whereas the second $\mathrm{Si}_{6}$ 
refers to the tetragonal bipyramid $\mathrm{Si}_{6}$. Moreover, in the paper IV (Ref. 8), we pointed out that another structural motif, namely, the fused-puckered-hexagonal-ring $\mathrm{Si}_{9}$ unit ${ }^{7,22,23}$ can be also a generic one for the low-lying clusters in the size range of $21 \leqslant n \leqslant 29$. Both the nine-atom motif $\left(\mathrm{Si}_{9}\right)$ and the six/six motif $\left(\mathrm{Si}_{6} / \mathrm{Si}_{6}\right)$ can be viewed as a portion of "adamantane" structure or a fragment of bulk diamond silicon. ${ }^{2,6}$

For neutral clusters in the size range of $27 \leqslant n \leqslant 40$, we previously reported that carbon fullerenes can be used as generic cage motifs to build endohedral fullerenelike lowenergy clusters. ${ }^{14}$ In that work, we determined the energy ordering (or the relative stability) of the clusters by using all-electron DFT calculation with the hybrid B3LYP functional along with the $6-311 \mathrm{G}(2 d)$ basis set. ${ }^{24}$ In this work, we reexamine the structures and relative stability of lowlying clusters in the size range of $31 \leqslant n \leqslant 40$ and $n=45$ by using the PBE (Ref. 25) and BLYP functionals. For those isomers with the lowest BLYP energy, we also optimized their structures by using all-electron DFT calculation with the hybrid B3LYP functional and the 6-31G $(d)$ basis set, followed by single-point calculation with the 6-311G $(2 d)$ basis set. The GAUSSIAN 03 package was used for the allelectron DFT calculation. ${ }^{26}$ The B3LYP calculations allow us to compare the newly obtained low-lying clusters with those previously obtained low-lying endohedral fullerene clusters. ${ }^{14}$ The low-energy nature of the reported clusters can be assessed by comparing the binding energies per atom of these candidate clusters with the predicted lowest-energy clusters in the size range of $21 \leqslant n \leqslant 30$. Again, we use the homologue carbon-fullerene cages to classify different families of isomers since this structural classification allows us to group many apparently different isomers into a single family.

\section{COMPUTATIONAL PROCEDURE}

To seek structures of medium-sized clusters with lower energy than previously obtained ${ }^{6,14,15}$ in the size range of $31 \leqslant n \leqslant 40$, we adopt a two-step search procedure: (1) an unconstrained search by using the minima-hopping $(\mathrm{MH})$ $\operatorname{method}^{27}$ coupled with a density-functional based tight-binding ${ }^{28}$ (DFTB) model of silicon and (2) geometry optimization of the top-ten lowest-energy isomers from the first step by using the plane-wave-pseudopotential DFT method with the PBE and BLYP functionals (implemented in the CPMD program ${ }^{29}$ ). The first step allows us to take advantage of global search of the potential energy surface (PES) described by the DFTB model and to obtain a database of candidate low-energy clusters for the second-step higher accuracy calculations. Since the DFTB model of silicon can describe the DFT PES more accurately than the previously used TB model and the Stillinger-Weber empirical model of silicon, ${ }^{14,15}$ more improved candidate isomers are expected from the first-step search, which may lead to clusters with lower energy in the second-step DFT calculation. The initial configurations for the first-step search were chosen from previous studies. ${ }^{6,14,15}$ In the second step, we performed geometry optimization for the top-ten lowest-energy isomers from the first step by using plane-wave-pseudopotential DFT method with the PBE and BLYP functionals. In the DFT
TABLE I. Calculated binding energy per atom for the low-lying isomers. The largest binding energies are in boldface.

\begin{tabular}{|c|c|c|c|}
\hline & \multirow{2}{*}{$\begin{array}{l}\text { Endohedral } \\
\text { fullerenes }\end{array}$} & \multicolumn{2}{|c|}{ Binding energy (eV/at.) } \\
\hline & & CPMD/PBE & CPMD/BLYP \\
\hline si31-1a & $\mathrm{Si}_{3} @ \mathrm{Si}_{28}$ & 3.913 & 3.339 \\
\hline $\operatorname{si3} 31-1 a^{\prime}$ & $\mathrm{Si}_{3} @ \mathrm{Si}_{28}$ & 3.894 & 3.343 \\
\hline si31-2 & & 3.881 & 3.345 \\
\hline si32-1a & $\mathrm{Si}_{4} @ \mathrm{Si}_{28}$ & 3.906 & 3.333 \\
\hline si32-1a' & $\mathrm{Si}_{2} @ \mathrm{Si}_{30}$ & 3.883 & 3.339 \\
\hline $\operatorname{si32}-1 a^{\prime \prime}$ & $\mathrm{Si}_{4} @ \mathrm{Si}_{28}$ & 3.905 & 3.338 \\
\hline si32-2 & & 3.885 & 3.353 \\
\hline si33-1a & $\mathrm{Si}_{3} @ \mathrm{Si}_{30}$ & 3.931 & 3.358 \\
\hline si33-2 & & 3.902 & 3.356 \\
\hline si34-1a & $\mathrm{Si}_{4} @ \mathrm{Si}_{30}$ & 3.934 & 3.351 \\
\hline si34-2 & & 3.910 & 3.356 \\
\hline $\operatorname{si35-1a}$ & $\mathrm{Si}_{5} @ \mathrm{Si}_{30}$ & 3.928 & 3.335 \\
\hline $\operatorname{si35-1a^{\prime }}$ & $\mathrm{Si}_{3} @ \mathrm{Si}_{32}$ & 3.917 & 3.355 \\
\hline si35-2 & & 3.898 & 3.354 \\
\hline si36-1a & $\mathrm{Si}_{4} @ \mathrm{Si}_{32}$ & 3.933 & 3.352 \\
\hline si36-1a' & $\mathrm{Si}_{4} @ \mathrm{Si}_{32}$ & 3.928 & 3.354 \\
\hline si36-1a" & $\mathrm{Si}_{4} @ \mathrm{Si}_{32}$ & 3.918 & 3.353 \\
\hline si36-2 & & 3.902 & 3.362 \\
\hline si37-1a & $\mathrm{Si}_{5} @ \mathrm{Si}_{32}$ & 3.940 & 3.357 \\
\hline si37-2 & & 3.915 & 3.362 \\
\hline si38-1a & $\mathrm{Si}_{4} @ \mathrm{Si}_{34}$ & 3.948 & 3.364 \\
\hline si38-2 & & 3.918 & 3.368 \\
\hline si39-1a & $\mathrm{Si}_{5} @ \mathrm{Si}_{34}$ & 3.939 & 3.365 \\
\hline si39-2 & & 3.909 & 3.361 \\
\hline si40-1a & $\mathrm{Si}_{6} @ \mathrm{Si}_{34}$ & 3.943 & 3.359 \\
\hline si40-1a' & $\mathrm{Si}_{4} @ \mathrm{Si}_{36}$ & 3.928 & 3.372 \\
\hline si40-2 & & 3.923 & 3.375 \\
\hline si45-1a & $\mathrm{Si}_{7} @ \mathrm{Si}_{38}$ & 3.956 & 3.375 \\
\hline $\operatorname{si45-1a^{\prime }}$ & $\mathrm{Si}_{5} @ \mathrm{Si}_{40}$ & 3.937 & 3.378 \\
\hline
\end{tabular}

optimization, we used the cutoff energy of 30 Ry for the plane-wave expansion and a supercell length of $25 \AA$. Note that although the energy ordering derived from the DFT calculations is different from the MH/DFTB search, we found that the structures of the isomers after geometry optimization are not much changed and that the lowest-energy isomer from the DFT calculation can be identified typically from one of the top-ten lowest-energy DFTB isomers. As in previous studies, ${ }^{6,14}$ we identified the corresponding homologue carbon-fullerene cages for the top-ten lowest-energy isomers in the second step.

\section{RESULTS AND DISCUSSIONS}

\section{A. Family I: Near-spherical endohedral fullerenelike clusters}

In Table I, we list the binding energies per atom of the lowest-energy clusters calculated by using PBE and BLYP functionals, respectively. The calculated total energies and the binding energies per atom using the B3LYP/6$311 \mathrm{G}(2 d)$ level of theory are given in Table II. The optimized structures of these lowest-energy clusters are displayed in Fig. 1, in which the endohedral atoms are highlighted in blue color and the outer cage in yellow color. The corresponding homologue carbon-fullerene cages are also 
TABLE II. Calculated total energy and binding energy per atom at the B3LYP/6-311G $(2 d)$ level of theory for the low-lying isomers. The geometries are optimized at the $\mathrm{B} 3 \mathrm{LYP} / 6-31 \mathrm{G}(d)$ level of theory. The isomers with the lowest total energy (or largest binding energy) and their energies are in boldface.

\begin{tabular}{|c|c|c|}
\hline & $\begin{array}{l}\text { B3LYP/6-311G(2d)// } \\
\text { B3LYP/6-31G }(d) \text { (a.u.) }\end{array}$ & $\begin{array}{c}\text { Binding energy } \\
\text { (eV/at.) }\end{array}$ \\
\hline si31-1a & -8975.0267101 & 3.343 \\
\hline si31-1a' & -8975.0394649 & 3.355 \\
\hline si31-2 & -8975.0397530 & 3.355 \\
\hline si32-1a & -9264.5370476 & 3.338 \\
\hline si32-1a' & -9264.5430933 & 3.343 \\
\hline si32-1a" & -9264.5478895 & 3.347 \\
\hline si32-2 & -9264.5657539 & 3.362 \\
\hline si33-1a & -9554.0875159 & 3.366 \\
\hline si33-2 & -9554.0856641 & 3.364 \\
\hline si34-1a & -9843.5965081 & 3.358 \\
\hline si34-2 & -9843.6159597 & 3.374 \\
\hline si35-1a' & -10133.1186964 & 3.362 \\
\hline si35-2 & -10133.1184408 & 3.362 \\
\hline si36-1a & -10422.6371317 & 3.363 \\
\hline si36-1a' & -10422.6387129 & 3.364 \\
\hline si36-1a" & -10422.643111 & 3.365 \\
\hline si36-2 & -10422.646323 & 3.370 \\
\hline si37-1a & -10712.1566624 & 3.364 \\
\hline si37-2 & -10712.1623258 & 3.368 \\
\hline si38-1a & -11001.6883421 & 3.374 \\
\hline si38-2 & -11001.6883437 & 3.374 \\
\hline si39-1a & -11291.2065318 & 3.374 \\
\hline si39-2 & -11291.1963501 & 3.367 \\
\hline $\operatorname{si40-1a^{\prime }}$ & -11580.7272519 & 3.376 \\
\hline si40-2 & -11580.7349497 & 3.381 \\
\hline si45-1a & -13028.3381654 & 3.388 \\
\hline $\operatorname{si4} 4-1 a^{\prime}$ & -13028.3396363 & 3.389 \\
\hline
\end{tabular}

displayed in Fig. 1 in gray color. Here, we use the notations $1 \mathrm{a}$ and $1 \mathrm{a}^{\prime}$ to name the cluster that has the lowest PBE and BLYP energies, respectively, in family I. For $\mathrm{Si}_{33}, \mathrm{Si}_{34}, \mathrm{Si}_{37}$, $\mathrm{Si}_{38}$, and $\mathrm{Si}_{39}$ it is found that the isomer which has the lowest PBE energy also has the lowest BLYP energy. Thus, only a single notation $1 \mathrm{a}$ is required to name their lowest-energy isomer in family I. Except $\mathrm{Si}_{36}, \mathrm{Si}_{37}, \mathrm{Si}_{39}, \mathrm{Si}_{40}$, and $\mathrm{Si}_{45}$, we found that the corresponding carbon-fullerene cages identified for the lowest-energy clusters are the same as those previously obtained using a different TB model or using a constrained basin-hopping/DFT search. ${ }^{14,30}$ In other words, the $\mathrm{MH} / \mathrm{DFTB}$ search only gives rise to slightly improved endohedral fullerene clusters with different configuration of the "stuffing" atoms but the same homologue fullerene cages. Detailed discussions for each size of clusters are given below.

\section{1. $\mathrm{Si}_{31}\left(\mathrm{Si}_{3} @ \mathrm{Si}_{28}\right)$}

As shown in Table I and Fig. 1, the lowest PBE-energy isomer si31-1a and the lowest BLYP-energy isomer si31-1a' have different structures. However, both have the same homologue carbon-fullerene cage $\mathrm{C}_{28}\left(T_{d}\right)$. We there-

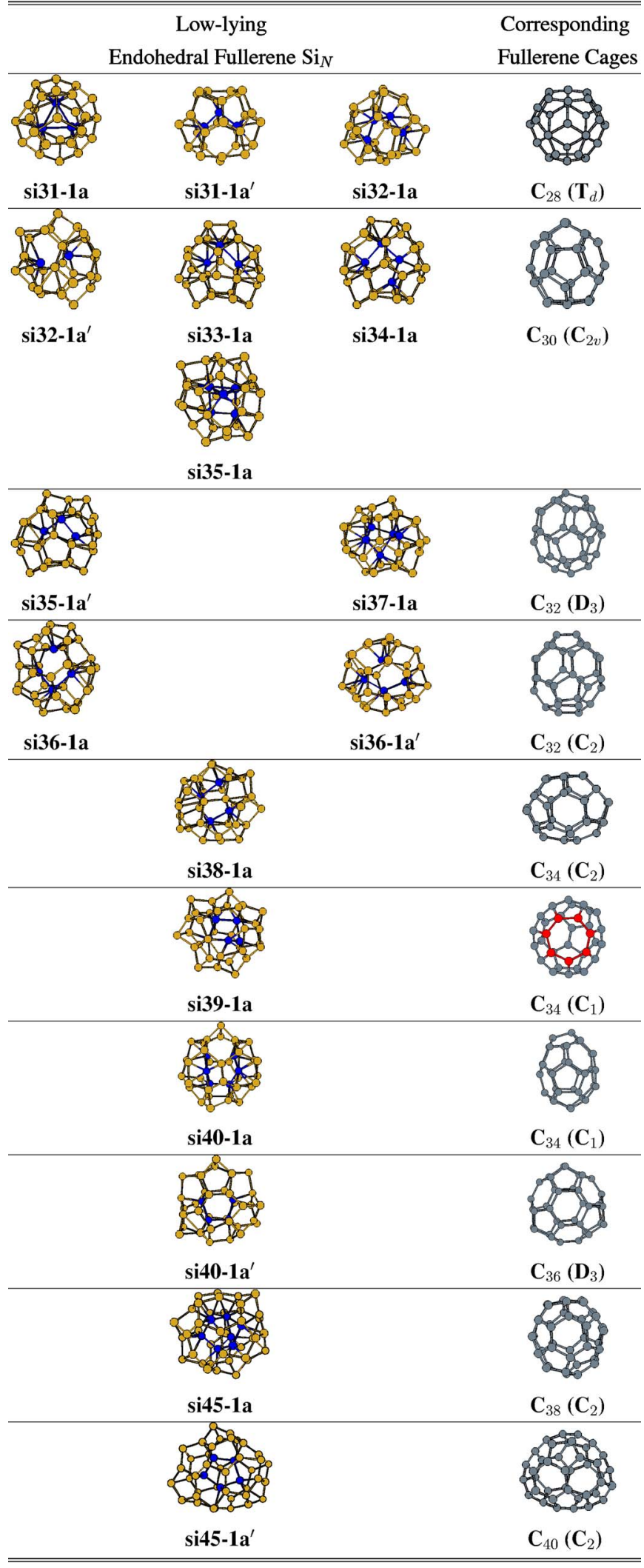

FIG. 1. (Color online) Geometries of the low-lying endohedral fullerenelike clusters of $\mathrm{Si}_{31}-\mathrm{Si}_{40}$ and $\mathrm{Si}_{45}$. The endohedral atoms are highlighted in blue color and the outer cage in yellow color. The corresponding homologue carbon-fullerene cages are shown in gray color and the seven-member ring in $\mathrm{Si}_{39}$ is highlighted in red color.

fore view them belonging to the same family. Note that si31-1a' is identical to the $\mathrm{Si}_{31}$ cluster reported in Ref. 14, as shown in Table II [B3LYP/6-311G(2d) total energy]. 


\section{2. $\mathrm{Si}_{32}\left(\mathrm{Si}_{4} @ \mathrm{Si}_{28}\right.$ or $\left.\mathrm{Si}_{2} @ \mathrm{Si}_{30}\right)$}

si32-1a is a new cluster and has four endohedral atoms while si32-1a' has two endohedral atoms and is identical to the $\mathrm{Si}_{32 \mathrm{a}}$ reported in Ref. 14. Although BLYP calculation indicates that si32-1a' is slightly lower in energy than si321a, the all-electron B3LYP/6-311G $(2 d)$ calculation indicates that si32-1a has a lower energy than si32-1a', as in the case of the PBE calculation. However, we found that $\mathrm{Si}_{32}$ reported in Ref. 14 has the lowest B3LYP/6-311G(2d) energy (Table II). Hereafter, we use the notation $1 \mathrm{a}^{\prime \prime}$ to name the cluster that has the lowest B3LYP/6-311G(2d) energy in family I. The si32-1a" has the same homologue carbon-fullerene cage $\mathrm{C}_{28}\left(T_{d}\right)$ as si32-1a.

\section{3. $\mathrm{Si}_{33}\left(\mathrm{Si}_{3} @ \mathrm{Si}_{30}\right)$}

si33-1a is new cluster which has a slightly lower B3LYP energy than the $\mathrm{Si}_{33}$ reported in Ref. 14, but both have the same homologue carbon fullerene cage $\mathrm{C}_{30}\left(C_{2 v}\right)$ and thus belong to the same family. The fact that si33-1a has the largest binding energy than any other $\mathrm{Si}_{33}$ isomers reported to date, regardless of the functional selected (PBE, BLYP, or B3LYP), and the fact that the simulated anion photoelectron spectra based on si33-1a are in excellent agreement with the experiment, ${ }^{6}$ render si33-1a the best candidate for the true global-minimum structure. We note that among mediumsized silicon clusters $(n>30), \mathrm{Si}_{33}$ and $\mathrm{Si}_{45}$ have received the most attention. ${ }^{9-12,14,15,31}$ This is largely because Smalley and coworkers have reported that these two clusters have unusually low chemical reactivity. ${ }^{32}$

\section{4. $\mathrm{Si}_{34}\left(\mathrm{Si}_{4} @ \mathrm{Si}_{30}\right)$}

si34-1a is a new cluster which has a slightly lower B3LYP energy than the $\mathrm{Si}_{34}$ reported in Ref. 14. However, both have the same homologue carbon-fullerene cage $\mathrm{C}_{30}\left(C_{2 v}\right)$ and thus belong to the same family.

\section{5. $\mathrm{Si}_{35}\left(\mathrm{Si}_{5} @ \mathrm{Si}_{30}\right.$ or $\left.\mathrm{Si}_{3} @ \mathrm{Si}_{32}\right)$}

Again, si35-1a' is a new cluster which has a slightly lower B3LYP energy than the $\mathrm{Si}_{35}$ reported in Ref. 14, but both have the same homologue carbon-fullerene cage $\mathrm{C}_{32}\left(D_{3}\right)$ and thus belong to the same family. PBE calculation, however, indicates that si35-1a is more likely to be the global minimum than si35-1 $\mathrm{a}^{\prime}$. It appears that the PBE functional favors more compact clusters with smaller cages whereas the BLYP functional favors more open clusters with larger cages.

\section{6. $\mathrm{Si}_{36}\left(\mathrm{Si}_{4} @ \mathrm{Si}_{32}\right.$ or $\left.\mathrm{Si}_{4} @ \mathrm{Si}_{32}\right)$}

si36-1a' is a new cluster which has a slightly lower BLYP energy but higher B3LYP/6-311G(2d) energy than the $\mathrm{Si}_{36}$ (or si36-1a") reported in Ref. 14. Both si36-1a and si36-1a' have four endohedral atoms and the same carbonfullerene cage $\mathrm{C}_{32}\left(C_{2}\right)$. However, the $\mathrm{Si}_{36}$ (or si36-1a") reported in Ref. 14 exhibits a different carbon fullerene cage $\mathrm{C}_{32}\left(D_{3}\right)$. We thus view si36-1a and si36-1a' belonging to the same family, different from si36-1a".

\section{7. $\mathrm{Si}_{37}\left(\mathrm{Si}_{5} @ \mathrm{Si}_{32}\right)$}

si37-1a is a new cluster which has an appreciably lower B3LYP energy than the $\mathrm{Si}_{37}$ reported in Ref. 14. This is in part because si37-1a has a different homologue carbonfullerene cage $\left[\mathrm{C}_{32}\left(D_{3}\right)\right]$ from the previous one $\left[\mathrm{C}_{34}\left(C_{2}\right)\right]$. Having the smaller cage and more endohedral atoms, si37-1a is more compact than the $\mathrm{Si}_{37}$ reported in Ref. 14 .

\section{8. $\mathrm{Si}_{38}\left(\mathrm{Si}_{4} @ \mathrm{Si}_{34}\right)$}

si38-1a is a new cluster which has a slightly lower B3LYP energy than the $\mathrm{Si}_{38}$ reported in Ref. 14. However, both clusters have the same homologue carbon-fullerene cage $\mathrm{C}_{34}\left(C_{2}\right)$ and thus belong to the same family.

\section{9. $\mathrm{Si}_{39}\left(\mathrm{Si}_{5} @ \mathrm{Si}_{34}\right)$}

si39-1a is entirely a new cluster which has a slightly lower B3LYP energy than the $\mathrm{Si}_{39}$ reported in Ref. 14. However, we found that the homologue carbon-fullerene cage of si39-1a contains a seven-member ring (highlighted in red color in Fig. 1) and thus is not a classical fullerene cage. The fact that si39-1a has an appreciably smaller (PBE) binding energy than its neighbor clusters (si38-1a and si40-1a) suggests that si39-1a is a relatively less stable cluster.

\section{0. $\mathrm{Si}_{40}\left(\mathrm{Si}_{6} @ \mathrm{Si}_{34}\right.$ or $\left.\mathrm{Si}_{4} @ \mathrm{Si}_{36}\right)$}

Both si40-1a and si40-1a' are new clusters with much lower energy than the $\mathrm{Si}_{40}$ reported in Ref. 14. In fact, both have different homologue carbon-fullerene cages from the previous one. The homologue carbon-fullerene cage for si40-1a is $\mathrm{C}_{34}\left(C_{1}\right)$ and that for si40-1a' is $\mathrm{C}_{36}\left(D_{3}\right)$ and thus they belong to different families. si40-1a was originally derived by Wang et al. (Ref. 16) via an exhaustive simulated annealing search, and it has the lowest PBE energy. Hence, again, it appears that the PBE functional favors more compact endohedral clusters with smaller cages whereas the BLYP functional favors more open endohedral clusters with larger cages.

\section{1. $\mathrm{Si}_{45}\left(\mathrm{Si}_{7} @ \mathrm{Si}_{38}\right.$ or $\left.\mathrm{Si}_{5} @ \mathrm{Si}_{40}\right)$}

We single out $\mathrm{Si}_{45}$ for clusters larger than $n=40$ because, as mentioned above, $\mathrm{Si}_{45}$ has been received particular attention $^{10-12,14,15,31}$ due to its unusually low chemical reactivity. ${ }^{32}$ Both si45-1a and si45-1a' are new clusters. The si45-1a has a slightly lower PBE energy than the two $\mathrm{Si}_{45}$ isomers reported in Refs. 14 and 15 but they all have the same homologue carbon-fullerene cage $\left[\mathrm{C}_{38}\left(C_{2}\right)\right]$ and thus belong to the same family. On the other hand si45-1a' belongs to a different family since the homologue carbonfullerene cage is $\mathrm{C}_{40}\left(C_{2}\right)$. Again, in this case, the PBE functional favors more compact clusters with smaller cages whereas the BLYP functional favors more open clusters with larger cages.

\section{B. Family II: "Handmade" Y-shaped three-arm clusters for $31 \leqslant n \leqslant 40$}

In Paper IV (Ref. 8), we showed that magic-clusterassembled medium-sized clusters can be energetically very 


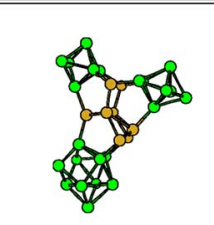

si31-2

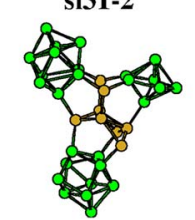

si35-2

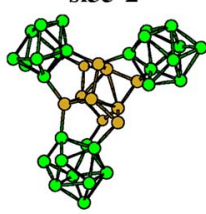

si39-2

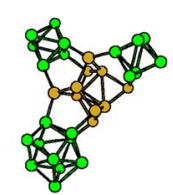

si32-2

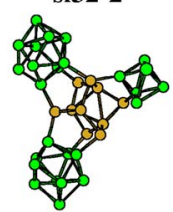

si36-2

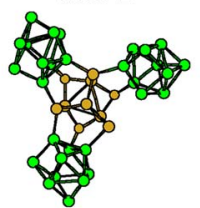

si40-2

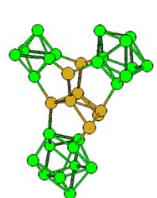

si33-2

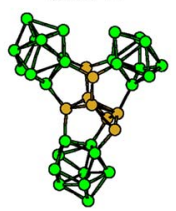

si37-2

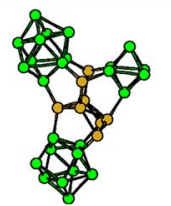

si34-2

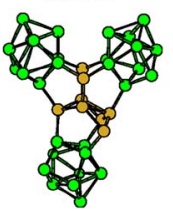

si38-2
FIG. 2. (Color online) Geometries of low-lying "Y-shaped three-arm" clusters $\mathrm{Si}_{31}-\mathrm{Si}_{40}$. The "glue part" is highlighted in yellow color and the three "arms" are in green color.

favorable in the size range of $\mathrm{Si}_{16}-\mathrm{Si}_{29}$, particularly when their total energies are calculated by using the BLYP functional. In that paper, we reported a new family of "handmade" clusters which are composed of a "glue unit" plus three magic clusters (from $\mathrm{Si}_{6}-\mathrm{Si}_{10}$ ). We call this hypothetical family of clusters the "Y-shaped three-arm" clusters. In Fig. 2, we display this family of clusters in the size range of $31 \leqslant n \leqslant 40$. Here, the glue units (the yellow-colored unit in Fig. 2) are very similar to the fused-puckered-hexagonal-ring $\mathrm{Si}_{9}$ unit but with one atom removed or added. The three "arms" (in green color) are various arrangements of the three magic-number clusters $\mathrm{Si}_{6}, \mathrm{Si}_{7}$, and $\mathrm{Si}_{10}$ and the TTP $\mathrm{Si}_{9}$. We performed geometry optimization by using the BLYP and B3LYP functionals for a limited number of clusters to find out the isomer with the lowest energy among various arrangements. Here, we use the notation 2 to denote the apparent lowest-energy isomers in family II. Their binding energies per atom calculated by using the BLYP and B3LYP functionals are listed in Tables I and II, respectively. Remarkably, it can be seen that except $\mathrm{Si}_{33}, \mathrm{Si}_{35}$, and $\mathrm{Si}_{39}$, the hand-made Y-shaped clusters all have lower energy than the endohedral fullerene clusters of family I, if the BLYP functional is selected. On the other hand, if the PBE functional is selected, the Y-shaped clusters are appreciably higher in energy than the endohedral fullerene clusters.

Smalley and co-workers ${ }^{32,33}$ performed photodissociation studies to neutral silicon clusters containing up to 60 atoms and found that medium-sized clusters larger than 30 atoms dissociate mainly by loss of the magic-number clusters $\mathrm{Si}_{10}$. It can be seen from Fig. 2 that the magic-number cluster $\mathrm{Si}_{10}$ dominates the population of the arm subunits, especially for the larger-sized clusters. In this sense, these low-lying Y-shaped three-arm neutral clusters provide an explanation to the photodissociation results for the mediumsized clusters beyond $n=30$.

\section{CONCLUSIONS}

We present two families of low-lying clusters in the size range of $30 \leqslant n \leqslant 40$, one is the endohedral fullerene family and another is the Y-shaped three-arm cluster family. The endohedral fullerene clusters have been systematically studied before and reported in Ref. 14. Here, we utilized an improved TB model of silicon so that we are able to obtain new low-lying clusters with slightly lower energy. Most of these new clusters belong to the same fullerene-cage family as previously reported since they have the same homologue carbon-fullerene cages. The binding energies of these lowlying clusters are all higher than those in the size range of $20 \leqslant n \leqslant 30$ reported. This result is consistent with the experiment by Jarrold and Honea ${ }^{34}$ that for clusters with 25 or more atoms, the dissociation (or binding) energies per atom increase rather smoothly.

Among the lowest-energy clusters obtained, we speculate that si33-1a is the best candidate for the true globalminimum structure. This is in part because si33-1a has the largest binding energy than any other $\mathrm{Si}_{33}$ isomers reported to date, independent of the functional selected (PBE, BLYP, or B3LYP). In addition, the simulated anion photoelectron spectra based on si33-1a are in excellent agreement with the experiment. ${ }^{6}$ For other clusters, because the lowest-energy cluster predicted based on the PBE functional differs from that based on the BLYP functional, the determination of the true global minimum will be quite difficult. However, as mentioned in the Introduction, we pay more attention in this study to the generic structural features of low-lying clusters as a function of size rather than to search a single (true) global-minimum structure. Toward this end, we use the homologue carbon-fullerene cage to classify different families of endohedral fullerene clusters, and we view those clusters with the same homologue carbon-fullerene cages and the same number of endohedral atoms belonging to the same family. This structural classification allows us to group many apparently different isomers into a single family. With this classification, we found that for $\mathrm{Si}_{31}, \mathrm{Si}_{33}, \mathrm{Si}_{36}, \mathrm{Si}_{37}, \mathrm{Si}_{38}$, and $\mathrm{Si}_{39}$ their lowest PBE- and BLYP-energy isomers belong to the same family.

We compared the binding energy per atom of a cluster (in Table I) with their two neighbor clusters and found that si32-1a (si32-1a') and si39-1a have notably smaller (PBE) binding energy than their neighbor clusters. These results suggest that for endohedral fullerene clusters in the size range of $30 \leqslant n \leqslant 40, \mathrm{Si}_{32}$ and $\mathrm{Si}_{39}$ are relatively less stable. In fact, we found that the homologue carbon cage of si39-1a has a seven-member ring and thus is not a classical fullerene cage.

Finally, we remark that most homologue carbonfullerene cages identified for the endohedral silicon fullerenes appear to be the most stable carbon cages among their IPR isomers. ${ }^{35}$ Here, the term "IPR isomers" refers to those isomers that satisfy the so-called isolated pentagon rule. ${ }^{36}$ In Table III, we provide the number of IPR carbon isomers as well as the symmetry of the isomers having the lowest DFTB energy. Clearly, by comparing Fig. 1 with Table III, one can see that many of the lowest-energy en- 
TABLE III. Calculated total energy of the lowest-energy IPR carbon fullerenes using the DFTB method.

\begin{tabular}{lccc}
\hline \hline $\mathrm{C}_{N}$ & $\begin{array}{c}\text { Total number of } \\
\text { IPR isomers }\end{array}$ & $\begin{array}{c}\text { Isomer with lowest DFTB } \\
\text { energy (a.u.) }\end{array}$ & $\begin{array}{c}\text { Point-group } \\
\text { symmetry }\end{array}$ \\
\hline $\mathrm{C}_{28}$ & 2 & -47.6249746 & $T_{d}$ \\
$\mathrm{C}_{30}$ & 3 & -51.0993035 & $C_{2 v}$ \\
$\mathrm{C}_{32}$ & 6 & -54.5983653 & $D_{3}$ \\
$\mathrm{C}_{34}$ & 6 & -58.0369625 & $C_{2}$ \\
$\mathrm{C}_{36}$ & 15 & -61.5161216 & $D_{2 d}$ \\
$\mathrm{C}_{38}$ & 17 & -64.979325 & $C_{2}$ \\
$\mathrm{C}_{40}$ & 40 & -68.445001 & $D_{2}$ \\
\hline \hline
\end{tabular}

dohedral silicon fullerenes and the carbon fullerenes (having the lowest DFTB energy) share the same fullerene cages, such as $\mathrm{C}_{28}\left(T_{d}\right), \mathrm{C}_{30}\left(C_{2 v}\right), \mathrm{C}_{32}\left(D_{3}\right), \mathrm{C}_{34}\left(C_{2}\right)$, and $\mathrm{C}_{38}\left(C_{2}\right)$.

\section{ACKNOWLEDGMENTS}

We are very grateful to valuable discussions with Professor K. A. Jackson, Professor B. C. Pan, Professor L.-S. Wang, Professor J. Zhao, and Professor J. Wang. This work is supported by grants from the DOE (DE-FG0204ER46164), the Nebraska Research Initiative, and by John Simon Guggenheim Foundation and the Research Computing Facility at University of Nebraska-Lincoln.

${ }^{1}$ M. F. Jarrold and V. A. Constant, Phys. Rev. Lett. 67, 2994 (1991).

${ }^{2}$ M. F. Jarrold, Science 252, 1085 (1991).

${ }^{3}$ M. F. Jarrold and J. E. Bower, J. Chem. Phys. 96, 9180 (1992).

${ }^{4}$ R. R. Hudgins, M. Imai, M. F. Jarrold, and P. Dugourd, J. Chem. Phys. 111, 7865 (1999)

${ }^{5}$ A. A. Shvartsburg, R. R. Hudgins, P. Dugourd, and M. F. Jarrold, Chem. Soc. Rev. 30, 36 (2001).

${ }^{6}$ J. Bai, L.-F. Cui, J. Wang, S. Yoo, X. Li, J. Jellinek, C. Koehler, T. Frauenheim, L.-S. Wang, and X. C. Zeng, J. Phys. Chem. A 110, 908 (2006)

${ }^{7}$ K. A. Jackson, M. Horoi, I. Chaudhuri, T. Frauenheim, and A. A. Shvartsburg, Phys. Rev. Lett. 93, 013401 (2004).

${ }^{8}$ S. Yoo and X. C. Zeng, J. Chem. Phys. 124, 054304 (2006).

${ }^{9}$ D. A. Jelski, B. L. Swift, T. T. Rantala, X. Xia, and T. F. George, J.
Chem. Phys. 95, 8552 (1991).

${ }^{10}$ U. Rothlisberger, W. Andreoni, and M. Parrinello, Phys. Rev. Lett. 72, 665 (1994).

${ }^{11}$ J. Pan and M. V. Ramakrishna, J. Chem. Phys. 101, 8108 (1994).

${ }^{12}$ M. Menon and K. R. Subbaswamy, Phys. Rev. B 51, 17952 (1995).

${ }^{13}$ Q. Sun, Q. Wang, P. Jena, S. Waterman, and Y. Kawazoe, Phys. Rev. A 67, 063201 (2003).

${ }^{14}$ S. Yoo, J. J. Zhao, J. L. Wang, and X. C. Zeng, J. Am. Chem. Soc. 126, 13845 (2004).

${ }^{15}$ J. Zhao, J. Wang, J. Jellinek, S. Yoo, and X. C. Zeng, Eur. Phys. J. D 34, 35 (2005)

${ }^{16}$ J. Wang, X. Zhou, G. Wang, and J. Zhao, Phys. Rev. B 71, 113412 (2005).

${ }^{17}$ S. Yoo and X. C. Zeng, J. Chem. Phys. 123, 164303 (2005).

${ }^{18}$ X. L. Zhu and X. C. Zeng, J. Chem. Phys. 118, 3558 (2003); Y. Gao, C. Killblane, and X. C. Zeng, Computing Lett. 1, 343 (2005)

${ }^{19}$ K.-M. Ho, A. A. Shvartsburg, B. Pan, Z.-Y. Lu, C.-Z. Wang, J. G. Wacker, J. L. Fye, and M. F. Jarrold, Nature (London) 392, 582 (1998).

${ }^{20}$ B. Liu, Z.-Y. Lu, B. Pan, C.-Z. Wang, K.-M. Ho, A. A. Shvartsburg, and M. F. Jarrold, J. Chem. Phys. 109, 9401 (1998)

${ }^{21}$ I. Rata, A. A. Shvartsburg, M. Horoi, Th. Frauenheim, K. W. M. Siu, and K. A. Jackson, Phys. Rev. Lett. 85, 546 (2000).

${ }^{22}$ S. Yoo and X. C. Zeng, Angew. Chem., Int. Ed. 44, 1491 (2005).

${ }^{23}$ O. Guliamov, L. Kronik, and K. A. Jackson, J. Chem. Phys. 123, 204312 (2005).

${ }^{24}$ A. D. Becke, Phys. Rev. A 38, 3098 (1988); J. P. Perdew, Phys. Rev. B 33, 8822 (1986); C. Lee, W. Yang, and R. G. Parr, ibid. 37, 785 (1988).

${ }^{25}$ J. P. Perdew, K. Burke, and M. Ernzerhof, Phys. Rev. Lett. 77, 3865 (1996).

${ }^{26}$ M. J. Frisch, G. W. Trucks, H. B. Schlegel et al., GAUSSIAN 03, Revision C.02, Gaussian, Inc., Wallingford, CT, 2004.

${ }^{27}$ S. Goedecker, J. Chem. Phys. 120, 9911 (2004).

${ }^{28}$ D. Porezag, Th. Frauenheim, Th. Köhler, G. Seifert, and R. Kaschner, Phys. Rev. B 51, 12947 (1995).

${ }^{29}$ J. Hutter, A. Alavi, T. Deutsch, M. Bernasconi, S. Goedecker, D. Marx, M. Tuckerman, and M. Parrinello, CPMD, Version 3.7.1, MPI für Festkörperforschung Stuttgart, 2001.

${ }^{30}$ D. J. Wales and H. A. Scheraga, Science 285, 1368 (1999).

${ }^{31}$ E. Kaxiras, Phys. Rev. Lett. 64, 551 (1990).

${ }^{32}$ J. L. Elkind, J. M. Alford, F. D. Weiss, R. T. Laaksonene, and R. E. Smalley, J. Chem. Phys. 87, 2397 (1987).

${ }^{33}$ Q. L. Zhang, Y. Liu, R. F. Curl, F. K. Tittel, and R. E. Smalley, J. Chem. Phys. 88, 1670 (1988).

${ }^{34}$ M. F. Jarrold and E. C. Honea, J. Phys. Chem. 95, 9181 (1991).

${ }^{35}$ C. Z. Wang, B. L. Zhang, and K. M. Ho, Computational Studies of New Materials (World Scientific, Singapore, 1999).

${ }^{36}$ H. W. Kroto, Nature (London) 329, 529 (1987). 